\title{
A Novel HPTLC Method for Simultaneous Quantification of (-)-Pseudosemiglabrin and Isoglabratephrin a Bioactive Prenylated Flavonoids Identified in Tephrosia Apollinea
}

Loiy Elsir Ahmed Hassan ${ }^{*}$, Mohd Shahrul Ridzuan Hamil ${ }^{2}$, Mohammed Ali Ahmed Saeed ${ }^{2}$ and Nornisah Mohamed $^{2}$

${ }^{1}$ Department of Pharmacology, School of Pharmaceutical Sciences, Universiti Sains Malaysia, Malaysia

${ }^{2}$ Department of Pharmaceutical Chemistry, School of Pharmaceutical Sciences, Universiti Sains Malaysia, 11800 Minden, Penang, Malaysia

\section{"Correspondence author}

Loiy Elsir Ahmed Hassan

Department of Pharmacology,

School of Pharmaceutical Sciences,

Universiti Sains Malaysia,

Malaysia

Submitted : 24 Aug 2020 ; Published : 22 Sep 2020

\begin{abstract}
Fingerprint analysis by HPTLC is an effective and powerful tool for linking the bioactive components profile of the plants with botanical identity and for estimation of chemical biomarkers. Tephrosia apollinea has been used in folk medicine to treat inflammation and relieving pain, as well as for diarrhea and abdominal pain. A densitometric High Performance Thin Layer Chromatography (HPTLC) fingerprinting method has been developed for the simultaneous determination and quantification of (-)-pseudosemiglabrin (SSG) and isoglabratephrin (IGP) in different parts of Tephrosia apollinea collected in summer and winter. The study revealed that SSG and IGP are the major components in T. apollinea and found in all parts of the plant at different amount. The proposed HPTLC method was found to be convenient for rapid screening of active components present in ethanol extracts of T. apollinea and can be used for analysis and routine quality control of the raw materials as well as formulations containing Tephrosia species.
\end{abstract}

Keywords: Tephrosia apollinea; HPTLC; Prenylated Flavonoids; (-)-Pseudosemiglabrin; Isoglabratephrin

\section{Introduction}

Medicinal herbs have a long history in curing various ailments and improving human health. In recent decade there has been a vast growth in the field of herbal medicine and these plant based drugs popularly are increasing both in developing and developed countries because of their natural origin, more therapeutic effect and less side effects. Due to intermixing of similar looking substances and different species of the same genera, it has become mandatory to find reliable characteristics ensuring the quality, safety, and efficacy of herbal drugs [1].

Tephrosia apollinea in annul shrub belong to family leguminaceae, its originated in northeast Africa and southwest Asia, in Sudan it distributed widely along the Nile valley, semi-arid areas northern Sudan and by the coast of the red sea [2]. T. apollinea has many pharmacological activities such as anti-inflammatory, antimicrobial, piscicidal, insecticidal, antiangiogneic and anti-cancer properties [3, 4]. The aerial parts of the plant used by traditional healers to treat inflammation associated with earache, bone fracture also it used to treat cough, bronchitis and wounds, recently we scientifically proof that the major phytoconstituent (-)-pseudosemiglabrin is responsible for anti-inflammatory and analgesic effect of the plant $[5,6]$. Isoglabratephrin was isolated from the aerial parts of $T$. apollinea in a bioassay guided approach as anticancer agent against prostate and pancreatic malignancies [4]; also this prenylated flavone has been identified as potent antifungal agent extracted from the same plant [7].

Fingerprint analysis by HPTLC is an effective and powerful tool for linking the bioactive components profile of the plants with botanical identity and for estimation of chemical and biochemical markers. On this background, an attempt has been made to establish chemical method for authentication and quantification of bioactive components in different parts of T. apollinea. Thus, a densitometry HPTLC analysis was performed for the development of characteristic fingerprint profile, which may be used as a marker for quality evaluation and standardization of the drugs. To the best of our knowledge this is the first qualitative and quantitative estimation of the major prenylated flavonoids (-)-pseudosemiglabrin and isoglabratephrin in different organs of T. apollinea aerial parts, roots and seeds. 


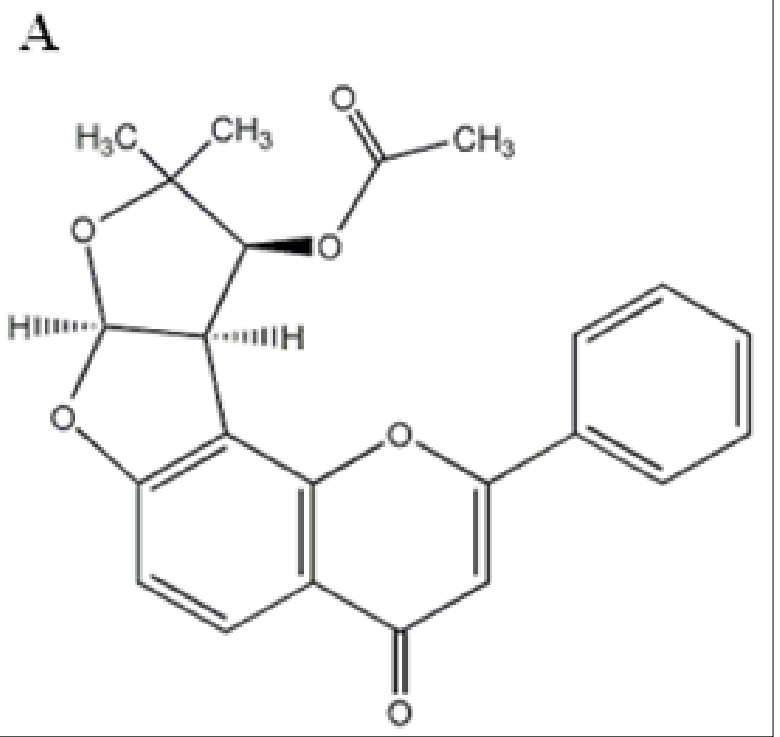

B

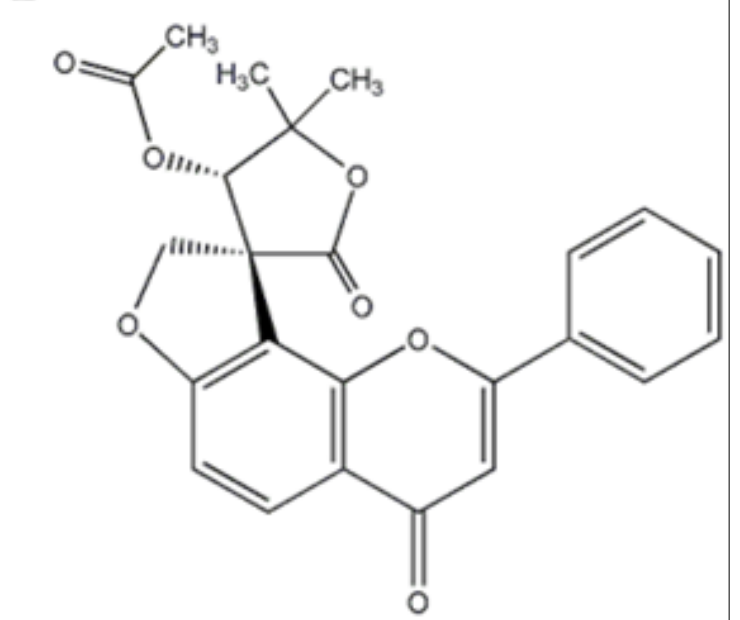

Figure 1: The structure of A) (-)-pseudosemiglabrin, B) isoglabratephrin

\section{Materials and methods}

\section{Reagents and standards}

All chemicals and solvents used were of analytical grade and obtained from Merck (Germany). Stock solutions of standards were prepared in methanol immediately before use. Solutions were applied using Linomat 5 applicator on TLC plates precoated with silica gel $60 \mathrm{~F} 254(20 \mathrm{x} 10 \mathrm{~cm}, 0.2 \mathrm{~mm}$ thick) obtained from Merck.

HPTLC Determination of bioactive compounds from $T$. apollinea

The two major prenylated flavonoid namely (-)-pseudosemiglabrin and isoglabratephrin were isolated from ethanolic extract of $T$. apollinea aerial parts in a bioassayguided fractionation studies and their chemical structures were identified by spectral analysis as well as by crystallography $[2,4]$.

Plant material

The plant T. apollinea collected from (Al-yasmin district)
Khartoum North in the month of September 2017. The plant was originally authenticated by Dr. W. Alsadig, a scientist and head of herbarium, Medicinal and Aromatic plants Research Institute (MAPRI) - National Center for Research, Sudan. A herbarium sample of this plant is preserved and deposited with MAPRI-R. A 1026-2017. The aerial parts were separated manually, air dried, powdered and saved in air tight container and subsequently referred to as aerial parts-summer. Seeds were separated from the dried fruits and divided into two parts; first part was grounded and kept in dried in -20C and the other part being cultivated in winter (21 November 2017 to 15 January 2018). The whole plant harvested and aerial parts separated from roots and seeds separated from the fruits, subsequently this batch referred as winter samples.

\section{Preparation of stock solutions}

(-)-Pseudosemiglabrin and Isoglabratephrin $(100 \mu \mathrm{g} / \mathrm{ml})$ were prepared by dissolving $100 \mu \mathrm{g}$ accurately weighed of each compound in $1 \mathrm{ml}$ methanol, then they were sonicated for $10 \mathrm{~min}$ to obtain the stock solution $(100 \mu \mathrm{g} / \mathrm{ml})$. The isolated compounds (reference standards) were prepared and mixed in methanol to give a mix standard solution with $10 \mu \mathrm{g} / \mathrm{ml}$ concentration.

\section{Preparation of plant extracts}

The dried powder materials of T. apollinea (aerial parts, roots and seeds collected in summer as well as in winter) were extracted by ethanol $(96 \%, 75 \%$ and $50 \%)$ using maceration method by soaking $10 \mathrm{~g}$ of plant material in $50 \mathrm{ml}$ ethanol for $48 \mathrm{~h}$. Extracts were concentrated under reduced pressure at $50-60^{\circ} \mathrm{C}$ and finally $1 \mathrm{mg}$ from each extract was dissolved in $1 \mathrm{~mL}$ methanol and filtered using Whatman filter paper number 1 to form a concentration of $1 \mathrm{mg} / \mathrm{ml}$.

\section{Chromatographic analysis and HPTLC profile}

HPTLC fingerprinting analysis was conducted using an HPTLC system (CAMAG, Switzerland) with a developed mobile phase system of chloroform: methanol (9.8:0.2). Pre-coated silica gel 60 F254 thin layer chromatography (TLC) plates $(20 \mathrm{~cm} \times 10$ $\mathrm{cm}$ ), layer thickness of $0.2 \mathrm{~mm}$ (E. Merck KGaA, Darmstadt, Germany) were used as stationary phase. Test and standard solutions were spotted as $5 \mathrm{~mm}$ band length on 60 F254 TLC plate using a CAMAG Linomat syringe and CAMAG Linomat 5 instrument. The plates were then developed in a pre-saturated chamber with the mentioned mobile phase system up to 90 $\mathrm{mm}$ in length of the plates. The plates were then air-dried and images at ultraviolet (UV) $254 \mathrm{~nm}$ and $366 \mathrm{~nm}$ were captured using CAMAG REPROSTAR 3 chamber. The densitometric analysis of the reference compounds were carried out using a CAMAG TLC scanner 3 in the absorbance mode at $310 \mathrm{~nm}$.

\section{Calibration curve SSG and IG}

The contents of marker compounds were determined using calibration curve established with a mixture of standard at concentration range from 10 to $100 \mathrm{ng} / \mathrm{band}$. A stock solution of mix standard compounds $(10 \mu \mathrm{g} / \mathrm{ml})$ was prepared in methanol. The different volumes of stock solution 1, 2, $4,6,8$ and $10 \mu 1$ were spotted on HPTLC plate to obtained 
concentration 10, 20, 40,60,80 and 100ng/band, respectively (band width $5 \mathrm{~mm}$, distance between tracks $7 \mathrm{~mm}$ ) using Linomat 5 applicator. Each concentration of peak area was plotted against the concentration of SSG and IG spotted. The linear regression of standard curve was determined with R2 \pm $\mathrm{SD}=0.9929 \pm 6.25 \% \& 0.9924 \pm 6.64 \%$. The linear regression line for is $\mathrm{y}=0.883 \mathrm{x}+11.475$ and $\mathrm{y}=1.109 \mathrm{x}+12.796(\mathrm{R} 2=$ $0.9929 \& 0.9924)$ for IG and SSG respectively (Fig. 2). The regression data have shown a good linear relationship over the concentration range of 10-100 ng/band. The linearity of calibration graphs and adherence of the system to Beer's law are validated by high value of correlation coefficient and the SD for intercept value is noticed to be less than $2 \%$ (RSD $0.28 \%$ ). No significant difference was observed in the slopes of standard curves (ANOVA; $\mathrm{p}<0.05$ ).
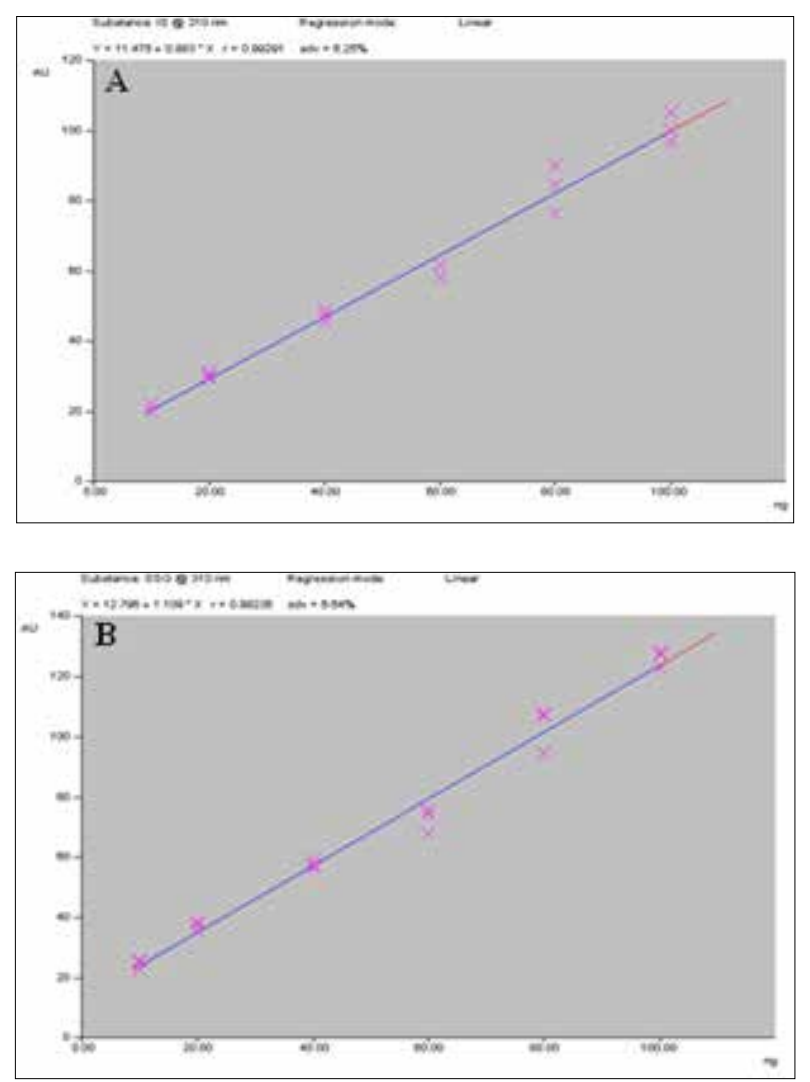

Figure 2: Calibration curve for standard compounds $(\mathrm{n}=6)$, A) isoglabratephrin, B) (-)-pseudosemiglabrin

\section{Detection and quantitation}

T. apollinea extracts solutions $(6 \mu \mathrm{Lof} 1 \mathrm{mg} / \mathrm{mL})$ were applied on TLC plates and subsequently developed as per chromatographic condition described in a glass tank pre-saturated with the mobile phase $(20 \mathrm{ml})$ chloroform: methanol $(9.8: 0.2)$ for $15 \mathrm{~min}$. The plates were developed at room temperature up to $90 \mathrm{~mm}$. After development, the plates were air dried and visualized under 254 and $366 \mathrm{~nm}$. Quantitative analyses of the compounds were evaluated by scanning the plates at $310 \mathrm{~nm}$ using CAMAG TLC scanner 3. The identification of SSG and IG were confirmed by superimposing the UV spectra of the samples and standards within same Rf 0.35 and 0.26 window, respectively. The analysis was repeated for 6 times and the possibility of interference from other components of extract in the analysis is studied. The spots at $\mathrm{Rf}=0.35$ and $\mathrm{Rf}=0.26$ corresponding to (-)-pseudosemiglabrin and isoglabratephrin were observed in the chromatogram of the extracts along with other components. There was no interference from other components present in the chromatogram (Figs. 3-5). The amount of SSG and IG present in the extracts were determined by fitting area values of the peak corresponding to the standards into their respective calibration curves. The results were presented in $\mathrm{ng} / \mathrm{band}(\mathrm{n}=3)$ (Table 2$)$.

\section{Statistical analysis}

Statistical calculations were carried out using SPSS 16.0 software package. Student's t-test was applied, and differences were considered significant at $\mathrm{P}<0.05$.

\section{Results}

In the current study quantitative estimation of biologically active prenylated flavonoids (-)-pseudosemiglabrin and isoglabratephrin components (Fig. 1) were conducted in different parts of $T$. apollinea using HPTLC. The two compounds represent the major phytoconstituents, therefore in the quantitative estimation it well represented in the chromatogram (Fig. 3 and Fig.5).

The mobile phase used in HPTLC analysis was selected by analyzing various compositions of different solvents in order to achieve high resolution and reproducible peaks. Of these, a mixture of chloroform and methanol (9.8: 0.2) was found to be the best mobile phase for development and analysis of (-)-pseudosemiglabrin and isoglabratephrin. TLC plate was observed under UV light $366 \mathrm{~nm}$ for the presence of SSG and IG which were detected with blue fluorescent spots; under UV light $254 \mathrm{~nm}$ IG exhibited bluish black spot, while SSG appeared as blue spot (Fig. 4). The developed HPTLC method gave an intense, compact and sharp peak of (-)-pseudosemiglabrin and isoglabratephrin at $\mathrm{Rf}=0.35$ and 0.26 respectively. The HPTLC images shown in Figs. 4 and Fig. 5 indicated that all sample parts were clearly separated without any tailing and diffuseness. This method was found to be very efficient in clearly separating the major biomarker compounds (SSG and IG) and other various constituents of $T$. apollinea with high resolution baseline, HPTLC flavonoids profile of $T$. apollinea ethanolic extracts was recorded in Fig. 5 and table 1. During the development of the HPTLC plate the optimized mobile phase volume for saturation was found to be $20 \mathrm{ml}$ and saturation time was found to be $15 \mathrm{~min}$. Densitometric analysis was carried out under UV $310 \mathrm{~nm}$. The Rf value ( 0.35 and 0.26$)$ for SSG and IG were similar in both samples as well as reference standards (Fig.3 and Fig 5). 

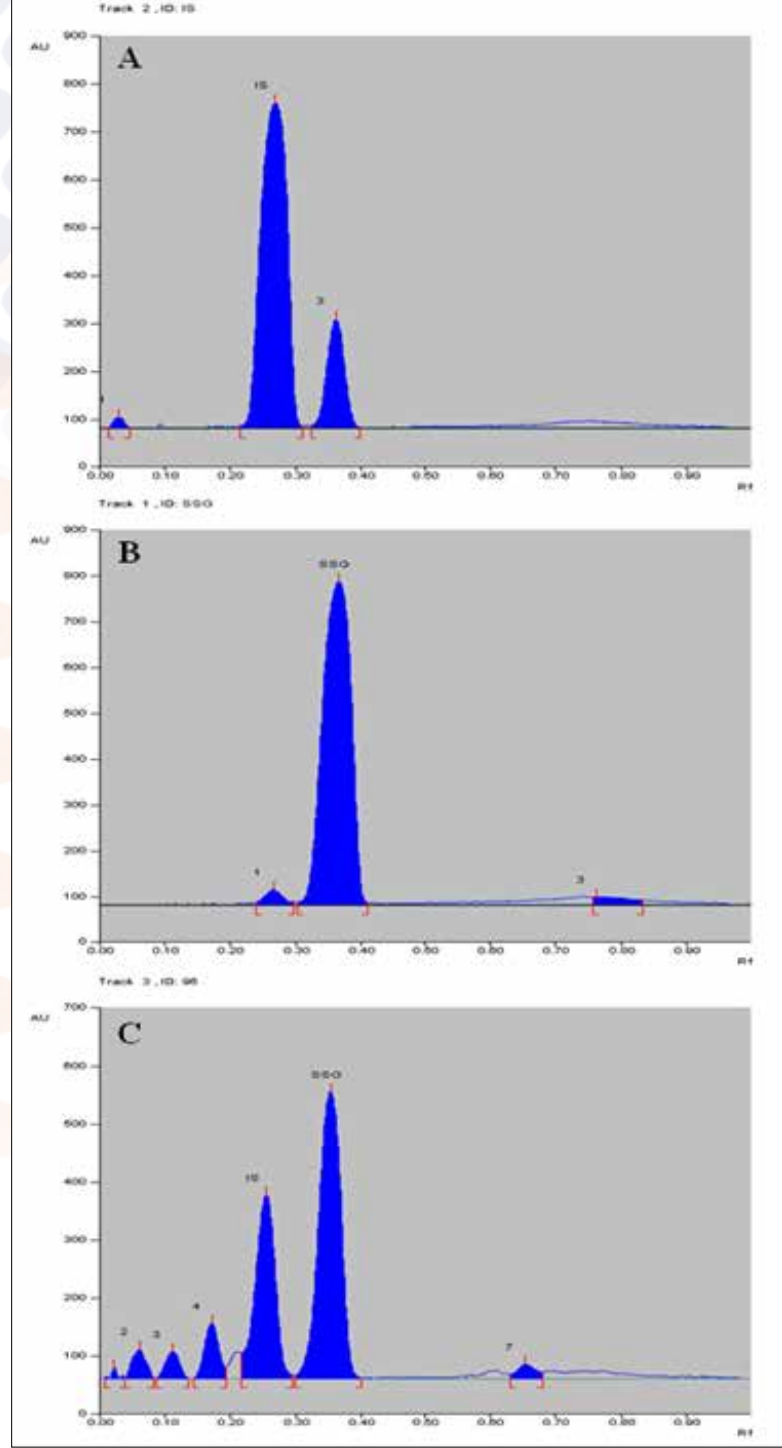

Figure 3: Peak response of A) standard isoglabratephrin, B) standard (-)-pseudosemiglabrin, C) (-)-pseudosemiglabrin and isoglabratephrin in the ethanol extract of $T$. apollinea

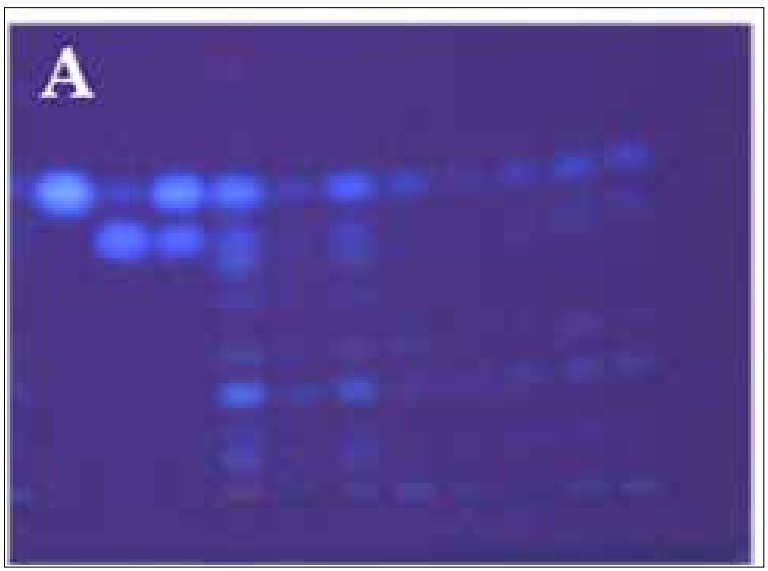

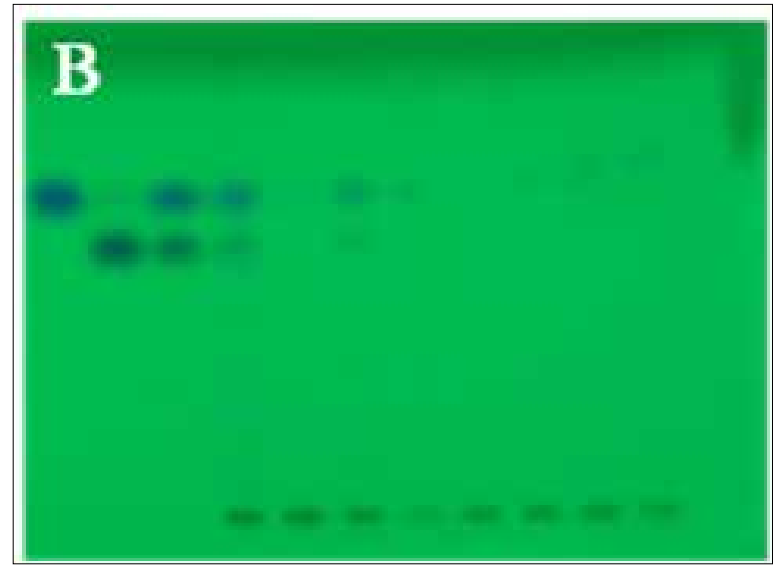

Figure 4: HPTLC chromatoplate of (-)-pseudosemiglabrin and isoglabratephrin along with ethanol extracts of $T$. apollinea different parts at A) $366 \mathrm{~nm}$ and B) $254 \mathrm{~nm}$. Lane 1(-)-pseudosemiglabrin, 2-isoglabratephrin, 3-mixture of markers, 4-11samples from T. apollinea different ethanolic extracts.
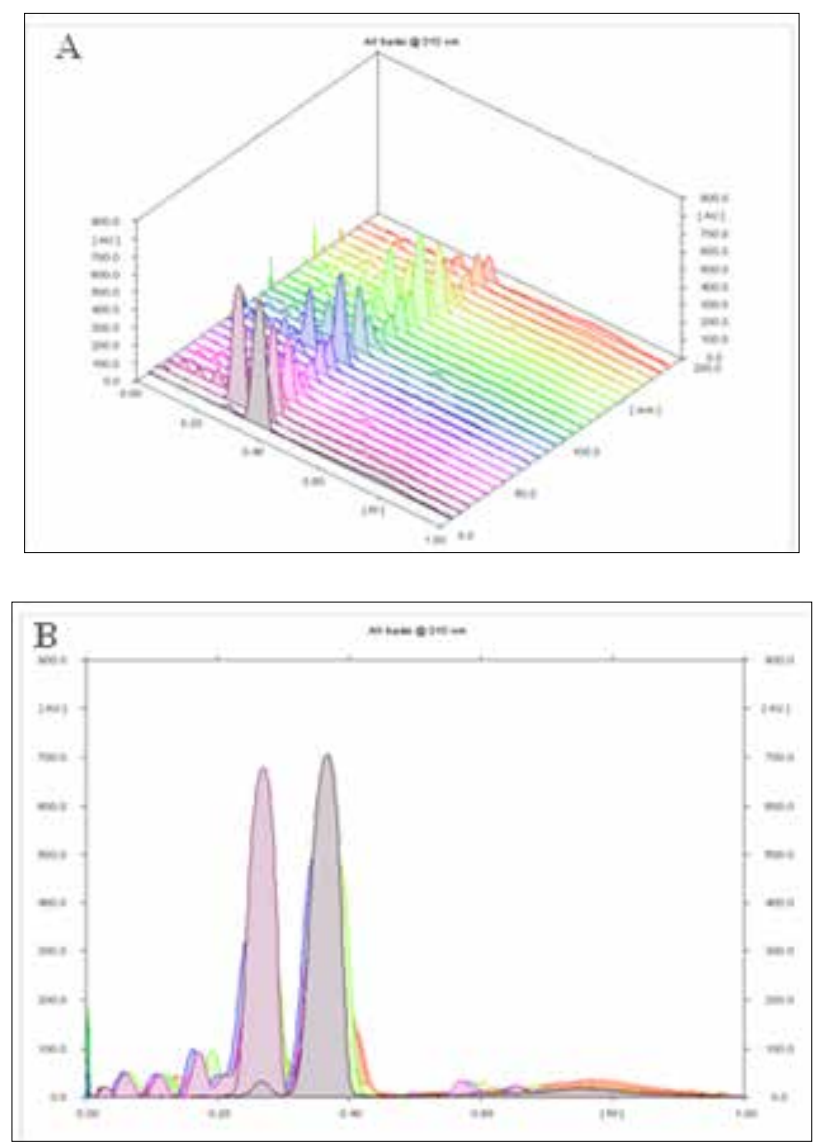

Figure 5: A) 3D Densitometric chromatogram of (-)-pseudosemiglabrin, isoglabratephrin and ethanol extracts of $T$. apollinea [aerial parts (ethanol 96\%,75\%,50\%), roots, aerial parts (summer), aerial parts (winter) seeds (summer) seeds (winter)] respectively. B) 2D Densitometric overlay Chromatogram of (-)-pseudosemiglabrin, isoglabratephrin and ethanol extracts of $T$. apollinea different organs. 


\begin{tabular}{|l|l|l|l|l|l|}
\hline Track & Peak & Rf & Height & Area & $\begin{array}{l}\text { Assigned } \\
\text { substance }\end{array}$ \\
\hline T3 & 1 & 0.02 & 18.9 & 139.8 & Unknown \\
\hline T3 & 2 & 0.06 & 50.7 & 984.4 & Unknown \\
\hline T3 & 3 & 0.11 & 46.3 & 919.2 & Unknown \\
\hline T3 & 4 & 0.17 & 94.9 & 1930.4 & Unknown \\
\hline T3 & 5 & 0.26 & 316.2 & 8903.2 & Isoglabratephrin \\
\hline T3 & 6 & 0.35 & 493.7 & 15520.1 & $\begin{array}{l}\text {-)- } \\
\text { pseudosemiglabrin }\end{array}$ \\
\hline T3 & 7 & 0.65 & 47.7 & 2.28 & Unknown \\
\hline
\end{tabular}

Table 1: Peak table with Rf values, height and area of prenylated flavonoids in T. apollinea ethanlic extract

Table 1 shows the profile of the two active prenylated flavonoids and other five unknown UV-sensitive compounds with retention factor (Rf) values between 0.02 and 0.65 . Both compounds showed highest peak areas compared to other compounds which confirm that these two active compounds are the major components in T. apollinea (Table 1) Fig. 3 and Fig.5. The two major prenylated flavonoids (SSG and IG) have been observed in T. apollinea different extracts with $\mathrm{Rf}$ value 0.35 and 0.26 respectively. In all parts of the plant SSG represents the highest content followed by IG, however SSG appears to be the only major phytoconstituent in the root extract with trace amount of other compounds including IG (Fig.6).

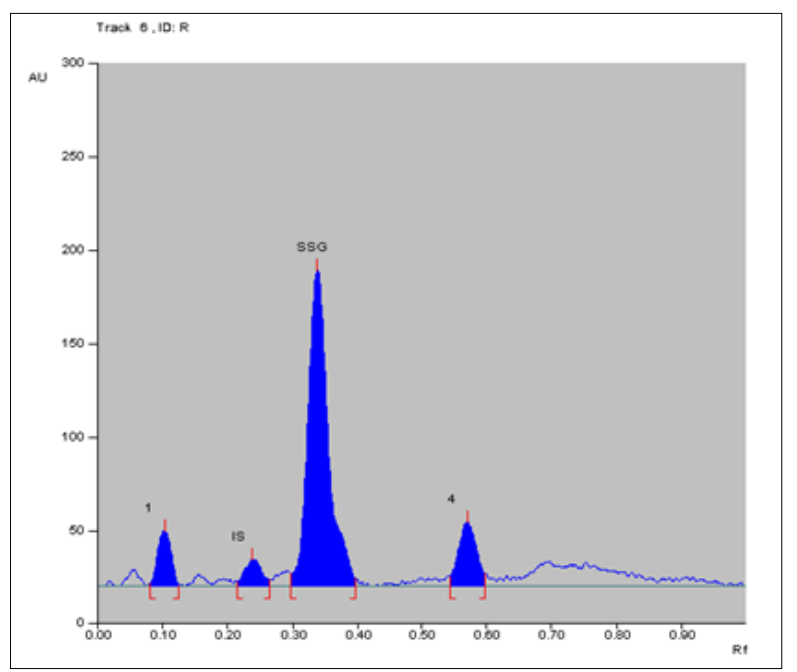

Figure 6: Peak response of (-)-pseudosemiglabrin and isoglabratephrin in T. apollinea root extract

A simple, accurate, and specific HPTLC method for simultaneous quantitative estimation of bioactive components in T. apollinea has been developed. The method employed in current study resulted in good peak shape of the maker compounds. The specific biologically active (-)-pseudosemiglabrin and isoglabratephrin were identified in different part of T. apollinea and thereby establishing the standard of those particular compounds for validation.

The application of developed HPTLC method was exercised in the estimation of SSG and IG in ethanol extracts of $T$. apollinea aerial parts, root and seeds collected in summer and winter. The contents of (-)-pseudosemiglabrin and isoglabratephrin quantified using TLC densitometric method were found higher in aerial parts collected during winter (more than two fold compared to those collected in summer), while no significant difference was observed between the seeds collected in summer and winter (Table 2). Furthermore, the contents of the marker compounds were estimated in the extracts of aerial parts obtained using different concentration of ethanol $(96 \%, 75 \%, 50 \%$ and $50 \%$ under $50^{\circ} \mathrm{C}$ ). By employing the above developed HPTLC the contents of (-)-pseudosemiglabrin and isoglabratephrin were found higher in $96 \%$ ethanol $(7.204 \pm 0.053 \%$ \& $5.839 \pm 0.09 \%)$ followed by $75 \%$ ethanol $(1.729 \pm 0.044 \% \& 0.891 \pm 0.011 \%)$ and $50 \%$ ethanol $(0.774 \pm 0.022 \% \& 0.285 \pm 0.025 \%)(\mathrm{w} / \mathrm{w})$ for SSG and IG respectively, however when applying some heat $\left(50^{\circ} \mathrm{C}\right)$ during maceration with 50\% ethanol the contents of SSG and IG significantly increased $(5.14 \pm 0.15 \%)$ and $(3.499 \pm 0.202 \%)$ respectively (Table 2).

\begin{tabular}{|l|l|l|}
\hline Sample & concentration (\%w/w) \\
\hline $\begin{array}{l}\text { Aerial parts } \\
\text { extract } \\
\text { (ethanol 96\%) }\end{array}$ & $7.203 \pm 0.052$ & $5.8391 \pm 0.106$ \\
\hline $\begin{array}{l}\text { Aerial parts } \\
\text { extract } \\
\text { (ethanol 75\%) }\end{array}$ & $1.729 \pm 0.044$ & $0.891 \pm 0.011$ \\
\hline $\begin{array}{l}\text { Aerial parts } \\
\text { extract } \\
\text { (ethanol 50\%) }\end{array}$ & $0.774 \pm 0.022$ & $0.285 \pm 0.028$ \\
\hline $\begin{array}{l}\text { Aerial parts } \\
\text { extract } \\
\text { (ethanol 50\%) } \\
\text { (Temperature } \\
\left.50^{\circ} \mathrm{C}\right)\end{array}$ & $5.14 \pm 0.15$ & $3.499 \pm 0.178$ \\
\hline Root extract & $2.297 \pm 0.056$ & $0.087 \pm 0.035$ \\
\hline $\begin{array}{l}\text { Aerial parts } \\
\text { (Summer) }\end{array}$ & $0.810 \pm 0.032$ & $0.311 \pm 0.015$ \\
\hline $\begin{array}{l}\text { Aerial parts } \\
\text { (Winter) }\end{array}$ & $1.454 \pm 0.062$ & $0.661 \pm 0.041$ \\
\hline $\begin{array}{l}\text { Seeds } \\
\text { (Summer) }\end{array}$ & $2.515 \pm 0.132$ & $1.319 \pm 0.072$ \\
\hline $\begin{array}{l}\text { Seeds } \\
\text { (Winter) }\end{array}$ & $2.158 \pm 0.301$ & $1.151 \pm 0.153$ \\
\hline
\end{tabular}

Table 2: Concentration of (-)-pseudosemiglabrin and isoglabratephrin in ethanol extracts obtained from different parts of T. apollinea

\section{Discussion}

Medicinal plants have historically considered as a reservoir of new leads for pharmaceutical development. The traditional medicine is an imperative resource of health care in developing countries, particularly in rural areas. The percentage of people dependent on botanical medicine for health care in Sudan is 
estimated at $90 \%$ [8]. Herbal remedies are extremely successful in curing chronic inflammatory disorders and relieving pain [6]. Herbal drugs are usually obtained as a mixture of more than one constituent, sometimes extract or fractionsrich in some specific phytoconstituents, and the efficacy of herbal drug depends on its phytochemical constituents [9]. Accurate identification and quality reassurance is an essential prerequisite for reproducible quality of herbal medicines [10]. Standardization is an imperative aspect for evaluating the quality and safety of herbal drugs. HPTLC Chromatographic fingerprint analysis emerged as a substantial tool for the qualitative and quantitative phytochemical analysis of the plant based drugs [11], it considered to be a feasible and rational approach for the quality assessment and species authentication of herbal medicine [12]. Thus, the objective of the present work was to develop HPTLC method for simultaneous estimation of two bioactive compounds namely (-)-pseudosemiglabrin and Isoglabratephrin. In previous study we proved scientifically that (-)-pseudosemiglabrin is the major phytoconstituent of $T$. apollinea responsible for anti-inflammatory and pain relief. It selectively inhibits cyclooxygenase-2 (COX-2) which is the isoenzyme responsible for production of prostaglandins in the inflamed tissues and mediate pain, with neglected effect on Cox1 (constitutive cytokine imperative for homeostatic functions, such as, in the gastric mucosa and platelets crucial for the integrity of intestine), also it manifested it efficacy by inhibiting pro-inflammatory cytokines TNF- $\alpha$ and IL-1in an in vitro settings as well as in vivo [6]. In previous studies (-)-pseudosemiglabrin and isoglabratephrin reported to have anticancer properties, they induce programmed cell death to a penal of cancer cell lines [2,4].

Several studies have shown that different organs of $T$. apollinea executed various medicinal properties, roots decoction is used to relieve constipation in children, as well as for treating colic pain and diarrhea [13]. It is reported that leaves and roots used for treatment of inflammation and pain associated with earache, bronchitis and bone fractures [6, 14]. The seeds are used as a paste for wounds treatment [5]. In the current study, quantitative estimation of specific biologically active components was conducted in the ethanol extracts obtained from aerial parts, roots and seeds of T. apollinea collected in summer and winter. The results provides useful agronomical information in term of the right season for cultivating the medicinal plants that can be used for herbal drug preparation, also the results gave clues about extraction techniques in order to obtain high amount of active principles. (-)-pseudosemiglabrin and isoglabratephrin are the major phytoconstituents in all organs of T. apollinea, however in the roots (-)-pseudosemiglabrin is the major principle, while isoglabratephrin and other compounds were found just in a tracing amounts, which may rationalize the popularity of using the roots by traditional healer for inflammatory disorder and relieving pain.

Standardization of herbal products promises constant composition of all of active principles responsible of pharmacological activities. Several researchers have proposed that HPTLC method enables high-quality resolution and can be used for quantification of biomarkers. The current study confirms such claims. The method can be used conveniently for the estimation of prenylated flavonoids (SSG and IG) in other species of Tephrosia (spp) as well as in herbal preparations and may be utilized for standardization purpose.

\section{Conclusion}

An accurate, rapid, simple and specific HPTLC method for quantitative estimation of bioactive principles present in Tephrosia apollinea has been developed. The method employed in current study resulted in good peak shape and enabled good resolution of SSG and IG from different part of plant materials. The essences of these active principles are beneficial for treating inflammation, pain, microorganism infections and combating cancers. The present standardization provides a specific and rapid tool in the herbal research, permitting to set quality specifications for identity transparency and reproducibility of biomarkers in herbal preparations of Tephrosia apollinea as well as other plants containing these bioactive prenylated flavonoids.

A comparative study of these active compounds has shown that it is present in higher amount in the aerial parts specifically collected during winter.

In summary, the proposed HPTLC method for the simultaneous analysis of (-)-pseudosemiglabrin and isoglabratephrin in different organs of Tephrosia apollinea is very simple, sensitive, economic and suitable for rapid routine quality control analysis and quantification of prenylated flavonoids in T. apollinea and other species and could be handy for standardization of herbal drugs.

\section{Acknowledgments}

The authors would like to thank the Ministry of Higher Education, Sudan (Grant no.328/2017) also we are thankful to the School of Pharmaceutical Sciences, Universiti Sains Malaysia for providing facilities and encouragement.

\section{References}

1. Ekor M (2013). The growing use of herbal medicines: Issues relating to adverse reactions and challenges in monitoring safety. Frontiers in Pharmacology, 4, Article 177.

2. Ahmed Hassan LE et al., (2014). Crystal structure elucidationand anticancerstudies of(-)-pseudosemiglabrin: A flavanone isolated from the aerial parts of Tephrosia apollinea. PLOS ONE, 9(3), p. e90806.

3. Nenaah GE (2014). Toxic and antifeedant activities of prenylated flavonoids isolated from Tephrosia apollinea $L$. against three major coleopteran pests of stored grains with reference to their structure-activity relationship. Natural Product Research, 28(24), 2245-2252.

4. Elsir A Hassan L et al., (2017). Colorectal, prostate and pancreas human cancers targeted bioassay-guided 
isolations and characterization of chemical constituents from Tephrosia apollinea. Anti-Cancer Agents in Medicinal Chemistry, 17(4), 590-598.

5. Ghazanfar SA and AM Al-Al-Sabahi (1993). Medicinal plants of Northern and Central Oman (Arabia). Economic Botany, 47(1), 89-98.

6. Hassan LEA et al., (2016). Evaluation of in vitro and in vivo anti-inflammatory effects of (-)-pseudosemiglabrin, a major phytoconstituent isolated from Tephrosia apollinea (Delile) DC. Journal of Ethnopharmacology, 193(Supplement C), 312-320.

7. Ammar MI, GE Nenaah, and A H H Mohamed (2013). Antifungal activity of prenylated flavonoids isolated from Tephrosia apollinea $L$. against four phytopathogenic fungi. Crop Protection, 49(Supplement C), 21-25.

8. Gomathi D et al., (2012). HPTLC fingerprinting analysis of Evolvulus alsinoides (L.) L. Journal of Acute Medicine, 2(3), 77-82.

9. Sheikh ZA et al., (2015). A novel HPTLC method for quantitative estimation of biomarkers in polyherbal formulation. Asian Pacific Journal of Tropical Biomedicine, 5(11), 955-959.

10. PatelDK, K Patel, and SP Dhanabal (2012). Standardization of Berberis aristata extract through conventional and modern HPTLC techniques. Asian Pacific Journal of Tropical Disease, 2(Supplement1), S136-S140.

11. Chawla R et al., (2013). Evidence based herbal drug standardization approach in coping with challenges of holistic management of diabetes: a dreadful lifestyle disorder of $21^{\text {st }}$ century. Journal of Diabetes \& Metabolic Disorders, 12(1), 35.

12. Kamboj A and AK Saluja (2017). Development of validated HPTLC method for quantification of stigmasterol from leaf and stem of Bryophyllum pinnatum. Arabian Journal of Chemistry, 10(Supplement1), S2644-S2650.

13. Aziz MA et al., (2018). Traditional uses of medicinal plants practiced by the indigenous communities at Mohmand Agency, FATA, Pakistan. Journal of Ethnobiology and Ethnomedicine, 14(1), 2.

14. Cheruth AJ et al., (2017). Medicinally active principles analysis of Tephrosia apollinea (Delile) DC. growing in the United Arab Emirates. BMC Research Notes, 10, 61. 